\title{
LATTICE SPACINGS FROM LATTICE FRINGES
}

\author{
P.G. SELF, H.K.D.H. BHADESHIA and W.M. STOBBS \\ Department of Metallurgy and Materials Science, University of Cambridge, Pembroke Street, Cambridge CB2 3QZ, England
}

Received 21 October 1980

\begin{abstract}
The different techniques available for the accurate measurement of lattice fringe spacings are discussed and their relative merit under different circumstances assessed. The relationship between the lattice fringe spacing and the lattice parameter is further examined for both uniform and chemically inhomogeneous material. The techniques described were also applied to an investigation of carbon segregation in residual austenite after the upper bainitic transformation in two steels.
\end{abstract}

\section{Introduction}

Modern transmission electron microscopes may be used routinely to obtain images with point-to-point resolution of well under $0.3 \mathrm{~nm}$ and axial lattice fringe images showing spacings of less than $0.2 \mathrm{~nm}$. However, in the majority of high resolution work the fine detail in the micrographs (such as absolute intensities and absolute fringe spacings) has not been fully exploited. Rigorous analyses have sometimes been completed successfully (e.g. O'Keefe and Buseck [1]) but in most cases full matching of fine detail with image calculations has proved impossible (e.g. Bursill, Barry and Hudson [2] and Anstis et al. [3]). These examples show that, unless the structural features of interest are relatively coarse compared with the attainable resolution, considerable care must be taken in the interpretation of images. Generally, even after full image simulation and quantitative matching of the computed and experimental micrographs, the structural features remain ambiguous. This is because of uncertainty in instrumental parameters $\left(C_{\mathrm{s}}, C_{\mathrm{c}}\right.$ etc.), the specimen thickness and also the very nature of electron scattering. Saxton [4] has reviewed some of the techniques which can be applied for the correction of artifacts in both linear and non-linear images.

A now quite generally applied method which promises material information with relative ease is that employed recently by Sinclair and Thomas [5] and reviewed by Sinclair [6]. It involves the measurement of lattice fringe spacings and their correlation with lattice spacing variation due to such effects as segregation. Our aim here is to delineate the accuracy of possible measurement methods and to indicate the factors which determine the accuracy of the technique when applied to different physical systems. At this stage it should be pointed out that the technique is complimented by the methods of convergent-beam electron diffraction $[7,8]$ which are of at least as high a potential accuracy in situations where a convergentbeam probe can be used on the specimen area of interest.

The relative accuracy of the various different methods for measuring spacings in a micrograph are described briefly in section 2 while the relative importance of the various ways in which such a measurement need not reflect the true lattice spacing is discussed in section 3. An example of the application of the method to the examination of the coarse segregation of carbon from upper bainitic ferrite to austenite in two steels, given in section 4 , is followed by a summary of our conclusions in section 5 .

\section{Fringe spacing measurement techniques}

There are several methods available for measuring lattice fringe spacings and it is not easy to tell which is the most accurate for a given system. The advan- 
tages and disadvantages of three techniques are summarised in this section. A feature common to any method is the need for a magnification calibration even if this is only indirect: an internal standard of known lattice spacing being available in the specimen of interest. If this is not the case the microscope magnification must be accurately determined as a function of specimen height using the objective lens current as a scale. In fact very small height changes can cause significant magnification changes. For example, using the short focal length JEOL $C_{\mathrm{s}}=0.7$ $\mathrm{mm}$ lens, a height change of only $10 \mu \mathrm{m}$ (as produced by a specimen tilt of one degree for a specimen 0.5 $\mathrm{mm}$ from the eucentric point of a specimen holder) gives as much as a $1 \%$ change in magnification.

\subsection{Moiré fringes}

A simple and quick way of determining fringe spacings is to compare the fringes with a ruled grating by forming moire fringes. The accuracy of the technique is generally insufficient for most applications, being limited both by the small number and breadth of the moiré fringes which can be used in the measurement. The usefulness of the method lies in the

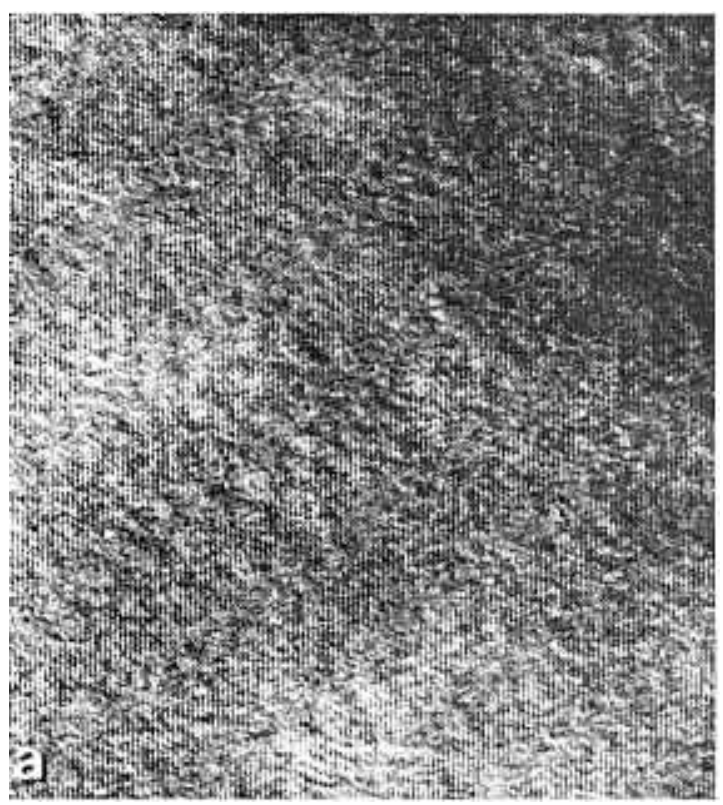

ease with which suitable areas for examination by more accurate methods can be located and micrographs giving irregular moiré fringes discarded.

If the spacing of lattice fringes is to be measured accurately, it is necessary to have large regions of fringes with uniform spacing. Any deviation from constant spacing may cause a significant error in the average fringe spacing obtained. Even micrographs of areas containing no crystallographic defects can often have changes in fringe orientation or spacing. This is in general associated with small changes in focus, specimen thickness and orientation across the specimen. Fig. 1 shows (111) fringes in aluminium. Although at first sight the fringes appear uniform the moiré fringes formed are curved. The largest regions which did not exhibit curved fringes were typically 300 fringe spacings in extent. Hence, in this particular case, although there were a thousand or so fringes on the micrograph, the accuracy is limited to that attainable using only 300 fringes.

A good way of applying the moiré technique is to project the micrograph onto a ruled grating. The typically low contrast levels of lattice fringe micrographs can be balanced to give optimum moiré fringe contrast by using a grating with dark to light line width

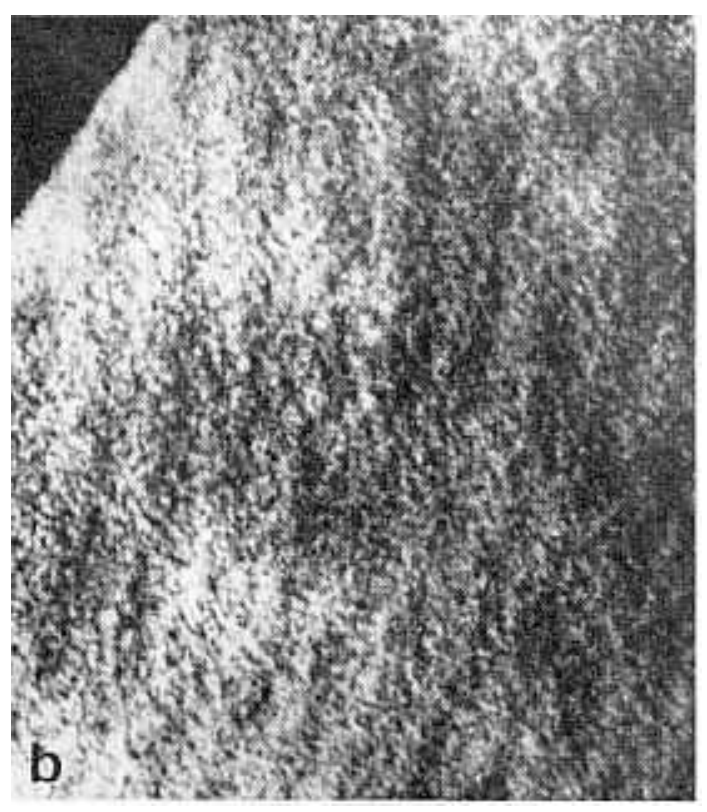

Fig. 1. An area of (111) fringes from aluminium (a) which appear straight yet which when compared with a ruled grating the resultant moiré fringes show a marked curvature (b). 
ratio of about 1 to 3 . Even so the moire fringes are difficult to record.

\subsection{Microdensitometry}

The principle of microdensitometric techniques is to count the number of fringes in a measured distance. This can be done in several ways using, for example, a travelling table microdensitometer, a travelling table microscope or computer processing. It is obviously essential that there are no defects along the measured line, and so a check of the micrograph by the moire method before proceeding with this often time consuming process is particularly useful.

Systematic error can be introduced into this technique by measuring along a line not perpendicular to the fringe direction. This can be eliminated by using a rotating table and measuring the fringe spacing for set misorientations. The true fringe spacing is then determined by finding the interpolated minimum value. Another source of systematic error can arise if the fringe shape is different at the two ends of the measured line, but this is usually detectable. With reasonable care the technique can be used to give the spacing of a set of fringes to an accuracy of $0.01 \%$ or better.

Another attractive technique under this general heading is to digitize the intensities on the micrograph and process them in a computer to give the fringe spacing using programs such as the SEMPER system [9]. The method is however time consuming and possible marginal improvements in accuracy are generally not warranted, given sources of error in the way the fringe spacing relates to that of the lattice.

In some applications it is necessary to measure the variation in spacing from fringe to fringe, as in the study of the metallurgically interesting phenomena of spinodal decomposition $[5,10]$. In this case the only suitable technique is microdensitometric. The accuracy is however then relatively low, mainly because of the difficulty in locating equivalent measuring points on each fringe - the contrast being locally variable.

\subsection{Optical transformation}

A standard method used in the measurement of spatial frequencies in a micrograph is to obtain its Fourier transform using an optical bench (for a review see Mulvey [11]). The technique's principal merit lies in the way an average for a large region is obtained directly giving potentially a higher accuracy than the other methods described. Even when examining small regions, provided they contain uniform fringes, the centres of the optically diffracted spots can be located with precision to give a value for the lattice fringe spacing to very high accuracy. Such measurements require, of course, that the optical bench conditions be optimised for spot measurement, and abberations in the optical system be minimized.

Experimentally the technique is most accurate when high-order spots can be used in the measurement. Reprinting the original micrograph onto high contrast film is sometimes advantageous (particularly if the original micrograph has low contrast) because the resultant squaring of the fringe contrast gives rise to higher-order spots. Generally, however, only second-order spots are produced in this way, mainly because of the marked variation in the width of the dark and light regions of a fringe along its length.

One advantage of the optical technique is that it is possible to have a defect in the area of the micrograph used to form the diffraction pattern and still get better accuracy than when examining a smaller defect-free region. This is provided that the defect is not associated with any fringe rotation except in a localised region of low contrast. The effect of defects on the optical diffractogram will vary from system to system, and so when measuring over a region known to contain a defect it is best to repeat the procedure for a defect-free region (necessarily of smaller size) to ensure that improved accuracy has in fact been obtained.

The above advantage of the technique also reflects its main disadvantage. This is that, although the average spacing of the fringes over the whole area examined may be found very accurately, the uncertainty in this value is difficult to determine. The only way of ascertaining the spread of spacing in the micrograph is by careful analysis of the diffracted spot intensities and shapes. In practice it is usually found that an examination of a through focal series best delineates the overall uncertainty in the measured fringe spacing (see section 3). Examples of typical diffractograms for systems varying from ideal to real structures are shown in fig. 2 . 

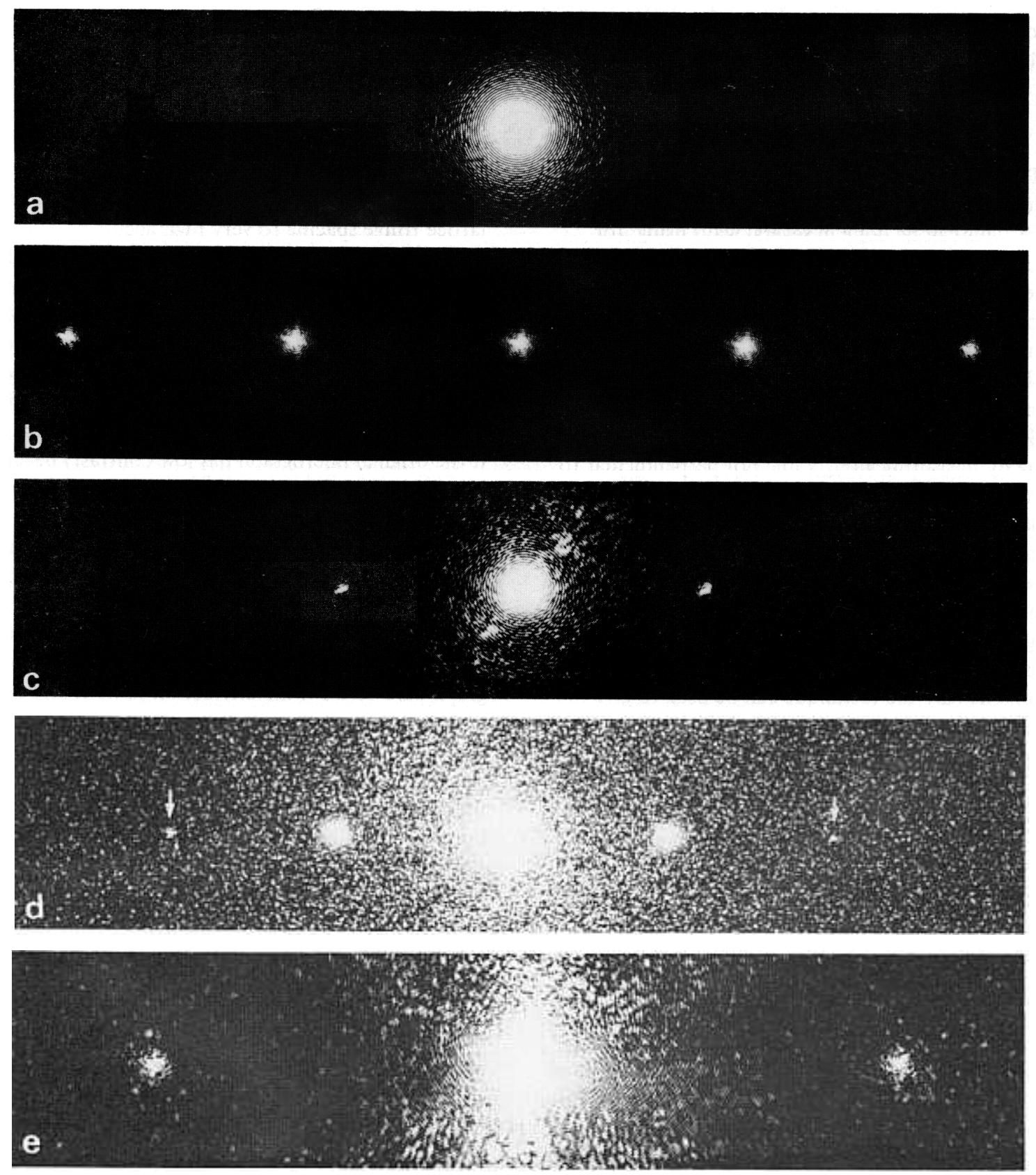

Fig. 2. Optical transforms for several systems: (a) the undiffracted beam, (b) a rule grating, (c) a micrograph with low contrast fringes, (d) contrast enhanced fringes and (e) a high defect density material.

\section{The relation between fringe spacing and lattice spacing}

Given that it is possible to measure lattice fringe spacings on a single micrograph to a measuring accuracy of $0.01 \%$ it is necessary to quantify how well the measured fringe spacing reflects the true lattice spacing. We have already noted some of the reasons 

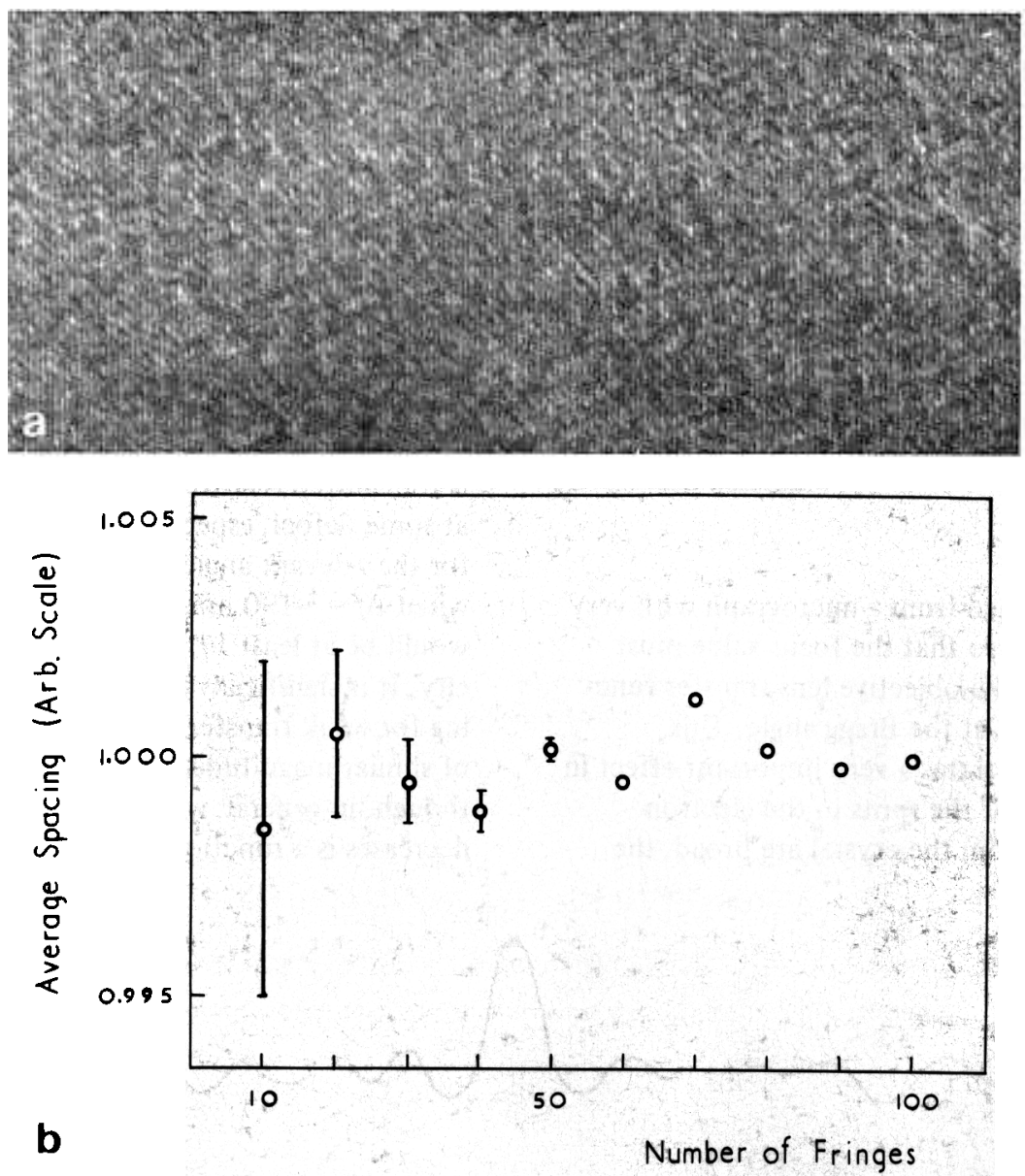

Fig. 3. The fringes from a high Chromium steel (a) and their spacing as measured on a travelling table microscope (b). As more fringes are counted the measuring accuracy increases but, as shown, the uncertainty in the true fringe spacing remains relatively high.

why the fringe spacing can vary locally from the true lattice spacing (see section 2.1). A single micrograph of even a perfect crystal will thus show variable spacings from point to point. It is this that will limit the accuracy of the fringe spacing measurement rather than the measuring accuracy of the technique used. This is clearly demonstrated in fig. 3 where spacing measurements are shown for a high purity $\mathrm{Fe}-\mathrm{Cr}-\mathrm{Ni}$ alloy. The plot shows that as the number of fringes used in the measurement is increased, and hence the measuring accuracy (indicated by the error bars) decreases, the spread in measured fringe spacing remains well above that to be expected from the measuring accuracy.
By considering the effects giving rise to local fringe spacing variation it becomes clear that regions showing variability will change as the objective lens transfer function is altered. Table 1 shows the results of fringe spacing measurements from a through focal series of the specimen area shown in fig. 3 . It can be seen that the variation in fringe spacing is much larger than that expected from the measuring error of any single micrograph which was $0.005 \%$. Hence in order to obtain a more realistic uncertainty in a lattice fringe measurement it is necessary to use the results obtained from a through focal series of the same region of specimen.

One of the results in table 1 is anomalously low. 


\section{Eable 1}

Finte spacings from a through focal series of a high thtomium steel; the theasurement starred is from a set of ringes with low contrast

$\begin{array}{ll}\text { Finge spacing } & \text { Average fringe spacing } \\ : 0.0002) & \text { (arbitrary units) }\end{array}$

\subsection{6}

1.7104

1.7094

$1.7095 \pm 0.0005(0.03 \%)$

$1.7078 *$

This value was obtained from a micrograph with very weak fringe contrast, so that the focus value must have been such that the objective lens transfer function was close to zero at the Bragg angle. This measurement demonstrates a very important effect in fringe measurement: if the spots in the electron diffraction pattern from the crystal are broad, the

resultant lattice fringe spacing can be very different from the lattice periodicity and change with the crystal tilt and defocus. It may even depend on spatial coherence. As an example, fig. 4 shows the amplitude profile of a diffraction spot broadened as it would be for a specimen area $20 \mathrm{~nm}$ in extent $(\operatorname{as}(\sin x) / x)$. The profiles are computed assuming a weak phase object for a $0.7 \mathrm{~mm} C_{\mathrm{s}}$ lens and a lattice of $0.4 \mathrm{~nm}$ if viewed axially or $0.2 \mathrm{~nm}$ if viewed with symmetrically tilted illumination. It may be seen that near to Scherzer defocus $(\sim-70 \mathrm{~nm})$ the amplitude is transferred relatively well but is severely distorted at some defoci, especially when the transfer function for the relevant angle is small. Note, for example, that when $\Delta f=-190 \mathrm{~nm}$ the fringe spacing observed would be at least $1 \%$ smaller than the lattice periodicity. It is significant that the observed change in spacing for weak transfer at moderate defocus (table 1) is of similar magnitude and sign to that predicted here though, in general, whether the spacing increases or decreases is a function of both lattice periodicity and
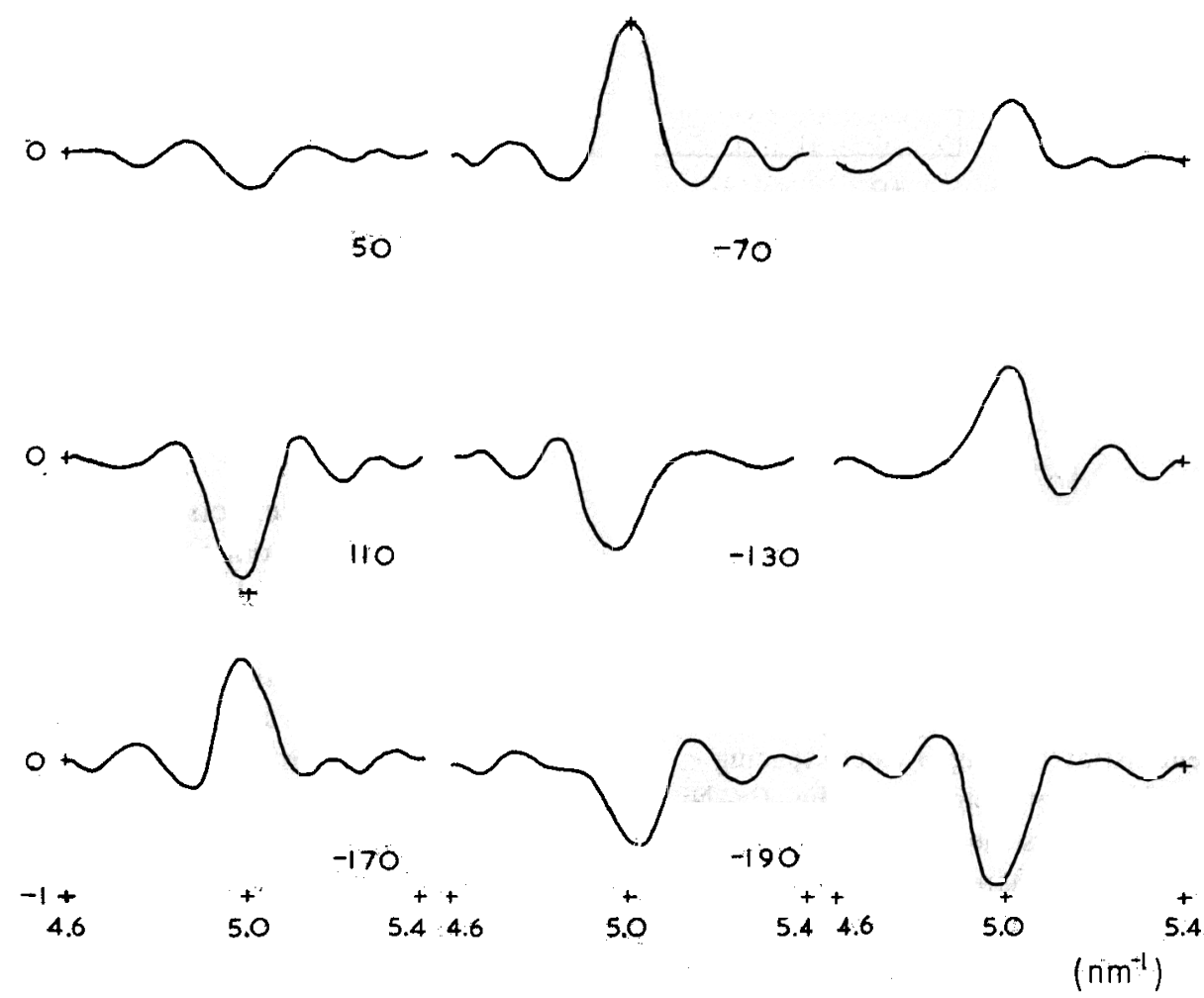

Fig. 4. The effect of lens transfer on a shape-broadened diffraction spot. The resultant spot shape is calculated at several defoci for a $C_{\mathrm{s}}=0.7 \mathrm{~mm}$ lens. The system is for a $20 \mathrm{~nm}$ area of $0.2 \mathrm{~nm}$ fringes with tilted illumination. 
lable 2

Fringe spacings from a through focal series of a spatially imited area of a $\mathrm{Cu}-\mathrm{Zn}-\mathrm{Al}$ alloy
Fringe spacing

$( \pm 0.002)$

Average fringe spacing

(arbitrary units)

2.210

2.221

2.209

2.203

2.221

2.207
$2.212 \pm 0.07(0.3 \%)$
2.216

defocus. These calculations also make it clear that the appropriate defocus for accurate transfer becomes more critical for smaller fringe spacing, especially if this is beyond the first zero of the transfer function.

The diffraction spots can be broad for a number of reasons. The most significant reason for broadening, and one which is always present irrespective of the system, is due to the specimen being of finite thickness. In conditions where the crystal is tilted away from the Bragg angle or the surface normal is at a high angle to the lattice planes, the fringe spacing will be markedly different from the true lattice periodicity. Table 2 shows the results of the fringe measurement (using a through focal series) from a spatially limited area of uniform fringes. The system was a martensitic memory alloy $(\mathrm{Cu}-\mathrm{Zn}-\mathrm{Al})$ of monoclinic structure and the fringes used were of approximately $0.6 \mathrm{~nm}$ spacing $\left(\frac{1}{3}(001)\right)$ and were observed in a region limited by two (001) plane stacking faults about $20 \mathrm{~nm}$ apart. The spread in the measured fringe spacing is some four times higher than the measuring error and again as would be expected with reference to the above calculation.

Just as when local irregular variations make the observed spacing sensitive to defocus we again conclude that to obtain a reasonable value of a lattice spacing it is necessary to measure a through focal series of the same region.

Situations in which observed fringe spacings have to be used even more carefully arise when the periodicity is locally variable. This is the case for spinodally decomposed systems. Spence et al. [12] have considered the effect of the lens transfer function for the $\mathrm{Au}-\mathrm{Ni}$ system examined by Gronsky and Thomas [13]. Spence et al. modelled the lattice periodicity as a sine function and showed that the local fringe spacing should vary with focus and that it can be drastically different (10-15\%) from the model spacing, even at optimum defocus. However, they were unable to explain the large spread in spacing observed by Gronsky and Thomas.

While it thus appears that the effect of the transfer function alone is sufficient to explain Gronsky and Thomas' result it is important to realise that Spence et al.'s arguments apply equally well to a system with uniform lattice spacing but sinusoidally varying projected lattice potential. Spinodal decomposition is of course associated with segregation and consequent changes in a local structure factor. These necessarily give rise to local variations in the amplitude and phase of the scattered waves. While the variable amplitude results in changes in contrast, phase variations necessarily cause a change in local fringe spacing. The effect can either increase or decrease the spacings observed on a local scale, though an increase would be generally expected in that higher atomic number elements normally segregate with associated increases in both lattice parameters and scattering factor.

Further problems include the fact that it is unlikely that spinodal decomposition is associated with a simple sinusoidal modulation of lattice spacing $[14,15]$. An effect of probably even greater importance is that the segregation in a thin foil will result in surface relaxations and further lattice distortions, exacerbated by the three-dimensional nature of segregation. This effect has been discussed by Cook and Howie [16] in another connection, and Howie [17] has recently pointed out its significance here.

Although the nature of all these effects is different the change required to allow for them in the twobeam analysis used by Spence et al. [12] is the same. Their equation for the two-beam intensity is

$$
I(x)=\left|\Phi_{0}\right|^{2}+2\left|\Phi_{0} \| \Phi_{u^{\prime}}(x)\right| \cos \left[2 \pi u_{x}^{\prime} x+\phi(x)\right]
$$

where $\Phi_{0}$ and $\Phi_{u^{\prime}}$ are the amplitude of the zeroth order and diffracted beam respectively. The local lattice spacing is $1 / u_{x}^{\prime}$ and $\phi(x)$ is the phase difference between the zeroth order and diffracted beam (including that introduced by the lens system). The 
spatial coordinate perpendicular to the fringes is $x$. All that is required to take into account the effects discussed above is to make the phase and amplitudes of the beams a function of $x$. The two-beam solution for the intensity becomes:

$$
\begin{aligned}
& I(x)=\left|\Phi_{0}(x)\right|^{2}+2\left|\Phi_{0}(x)\right|\left|\Phi_{u^{\prime}}(x)\right| \\
& \quad X \cos \left[2 \pi u_{x}^{\prime} x+\phi(x)\right] .
\end{aligned}
$$

This is more difficult to analyse than the original expression and a full solution would require both careful choice of model and comprehensive machine analysis. However, the important effects on the fringe profiles can be deduced intuitively.

Consider, for example, two adjacent areas with uniform but different lattice spacing. These areas will give rise to different scattered amplitudes and phases. Both will show uniform fringe spacings but the fringes will be shifted relative to the local lattice plane positions differently in each area. The interface between the two regions will thus show a large discrepancy in the local fringe spacings. Examination of the fringe profiles reported by Gronsky and Thomas [13] do indeed suggest that the major anomaly is the grouping of a few fringes of extremely small spacing as the above qualitative argument would indicate remembering that the discrepancy will be further compounded by the effects of defocus.

On the same approach it is clear that as the objective lens focus is changed the regions where the fringes are more uniformly spaced will show little change in spacing but rather a bulk shift of the fringes. At the modelled block interface the fringe spacing could vary quite markedly. This places doubt on the practice of averaging over a few fringe spacings to increase accuracy, but on the other hand suggests an improvement to the method for determining local lattice spacings. If the fringe profiles are plotted for each micrograph of a through focal series of images the local regions where the fringe spacing stays relatively constant should more accurately reflect the local lattice spacings. Gronsky and Thomas' fringe profile thus suggest the importance of including nonsinusoidal terms in the modelled expressions for the spacings. Appropriate models could probably thus be found by comparison of dark-field images as a function of deviation parameter.

\section{Application of the method to carbon segregation in two steels}

In this section we examine the accuracy of the technique when applied to a real problem: the segregation of carbon from ferrite to austenite in two steels. The compositions of the two steels examined were (in wt\%):

Alloy 1: $3 \% \mathrm{Mn}, 2.02 \% \mathrm{Si}, 0.43 \% \mathrm{C}$ and remainder $\mathrm{Fe}$, Alloy 2: $4.08 \% \mathrm{Ni}, 2.05 \% \mathrm{Si}, 0.39 \% \mathrm{C}$ and remainder Fe.

The former alloy was austenitised at $1100^{\circ} \mathrm{C}$ for $10 \mathrm{~min}$, isothermally transformed at $350^{\circ} \mathrm{C}$ for 1000 min and then water quenched. The latter material was similarly austenitised but isothermally transformed at $340^{\circ} \mathrm{C}$ for $60 \mathrm{~min}$ before water quenching. These heat treatments place the alloys in the upper bainite transformation temperature range and the time periods of the isothermal treatments ensure reaction termination in both cases [18].

The bainite transformation in steels is a displacive reaction $[19,20]$ in which the ferrite $(\alpha)$ is initially supersaturated with respect to carbon. In the absence of carbide precipitation (prevented in the present steels by the use of silicon), the excess carbon in the bainitic ferrite is subsequently rapidly partitioned into the residual austenite $(\gamma)$. The further transformation of this residual austenite to bainitic ferrite ceases when the carbon content of the former reaches a level $x_{\gamma}\left(T_{0}\right)$, such that displacive transformation becomes thermodynamically impossible [20]. Although such austenite can no longer transform to bainite, it can-continue to accumulate carbon from suitable sources up to a limit given by the no-substitutional partitioning $A e_{3}^{\prime}$ curve [18-20], i.e. $x_{\gamma}\left(A e_{3}^{\prime}\right)$. Such circumstances arise naturally during bainite sheaf formation [20] when a region of austenite, which has been affected by the dumping of carbon from an extant bainite plate, becomes isolated by the formation of new supersaturated bainite plates in its close proximity. The subsequent partitioning of carbon from these initially supersaturated bounding plates can raise the carbon content of the entrapped austenite film to any level within the range $x_{\gamma}\left(T_{0}\right) \leqslant$ $x_{\gamma} \leqslant x_{\gamma}\left(A e_{3}^{\prime}\right)$. The lower limit arises from the fact 
Table 3

Experimental results for the carbon content $\left(x_{\gamma}\right)$ of retained austenite in two steels (see text), the second column indicates the number of micrographs in the through focal series used in the measurement; note the marked variation in carbon content from region to region in both alloys

\begin{tabular}{|c|c|c|c|c|c|}
\hline \multirow[t]{2}{*}{ Region } & \multirow{2}{*}{$\begin{array}{l}\text { Number } \\
\text { in series }\end{array}$} & \multicolumn{2}{|c|}{$d(111)_{\gamma} / d(011)_{\alpha}$} & \multicolumn{2}{|l|}{$x_{\gamma}(w t \%)$} \\
\hline & & Micro. & Optical & Micro. & Optical \\
\hline \multicolumn{6}{|l|}{ Alloy 1} \\
\hline $\begin{array}{l}2 \\
3\end{array}$ & $\begin{array}{l}6 \\
4 \\
5\end{array}$ & $\begin{array}{l}1.029 \pm 0.001 \\
1.033 \pm 0.002 \\
1.046 \pm 0.002\end{array}$ & $\begin{array}{l}1.028 \pm 0.001 \\
1.034 \pm 0.001\end{array}$ & $\begin{array}{l}1.31 \pm 0.08(6 \%) \\
1.62 \pm 0.16(10 \%) \\
2.66 \pm 0.16(6 \%)\end{array}$ & $\begin{array}{l}1.23 \pm 0.08(7 \%) \\
1.70 \pm 0.08(5 \%)\end{array}$ \\
\hline Predicted & & $1.023-1.047$ & $.023-1.047$ & $0.85-2.80$ & $0.85-2.80$ \\
\hline \multicolumn{6}{|l|}{ Alloy 2} \\
\hline $\begin{array}{l}1 \\
2 \\
3 \\
4\end{array}$ & $\begin{array}{l}3 \\
4 \\
5 \\
3\end{array}$ & $\begin{array}{l}1.030 \pm 0.003 \\
1.040 \pm .0 .005 \\
1.044 \pm 0.001 \\
1.076 \pm 0.002\end{array}$ & & $\begin{array}{l}1.39 \pm 0.24(17 \%) \\
2.18 \pm 0.40(18 \%) \\
2.50 \pm 0.08(3 \%) \\
5.06 \pm 0.16(3 \%)\end{array}$ & \\
\hline Predicted & & $1.028-1.053$ & $1.028-1.053$ & $1.25-3.20$ & $1.25-3.20$ \\
\hline
\end{tabular}

that any austenite with a carbon content less than $x_{\gamma}\left(T_{0}\right)$ should ultimately transform to bainite. It should be noted that although substitutional alloying elements do not partition during the bainite transformation, their influence on the free energies of austenite and ferrite is manifested in the $x_{\gamma}\left(T_{0}\right)$ and $x_{\gamma}\left(A e_{3}^{\prime}\right)$ values. Thus any experimental measurements should reflect generally higher carbon levels in the case of alloy 2 (see table 3). Accordingly the lattice parameters of the retained austenite in each alloy will be different. On the other hand, since the final carbon content of the bainitic ferrite is very small and does not vary significantly, the lattice parameter of the ferrite can be used as an internal relative standard for both the sets of austenite lattice fringe spacing measurements.

The results described below thus test both the thermodynamic approach used and the predictions of the previous sections on the relationship between lattice fringe and lattice spacings. The two steels were specifically chosen to have very different predicted austenite carbon content limits in order better to test the technique of estimating $x_{\gamma}$ by lattice fringe measurements,

\subsection{Results and discussion}

For the reasons discussed above, the ferrite in both the alloys should have a constant lattice parameter of $0.28664 \mathrm{~nm}$ [21]. Although two distinct experimental relationships have been described for the lattice parameter of austenite as a function of carbon content $[22,23]$, it seems that the approach of Roberts [22] is more appropriate in the current application [18]. His expression for the austenite lattice parameter, $a_{\boldsymbol{\gamma}}$ is:

$$
a_{\gamma}=0.3555+0.0044 x_{\gamma}(\mathrm{nm}) \text {, }
$$

where $x_{\gamma}$ is the wt\% of carbon in austenite.

Images from each material are shown in fig. 5 and each exhibits both $(011)_{\alpha}$ and $(111)_{\gamma}$ lattice fringes. Qualitatively the defect density is high in both materials and is apparently significantly higher in the austenite of alloy 2 which had a lower volume fraction of this phase. Correspondingly, the application of convergent-beam techniques was impracticable and the application of lattice fringe measurement techniques is suitably difficult. In fact, the lattice fringe spacing of the retained austenite in alloy 2 could not be measured by the optical technique.

The results from several through focal series for a number of different regions of each alloy are given in table 3 . Here, since the measurements made were relative, only the ratios for the $(011)_{\alpha}$ and $(111)_{\gamma}$ spacings are shown. Absolute values may be determined using the ferrite lattice parameter and 

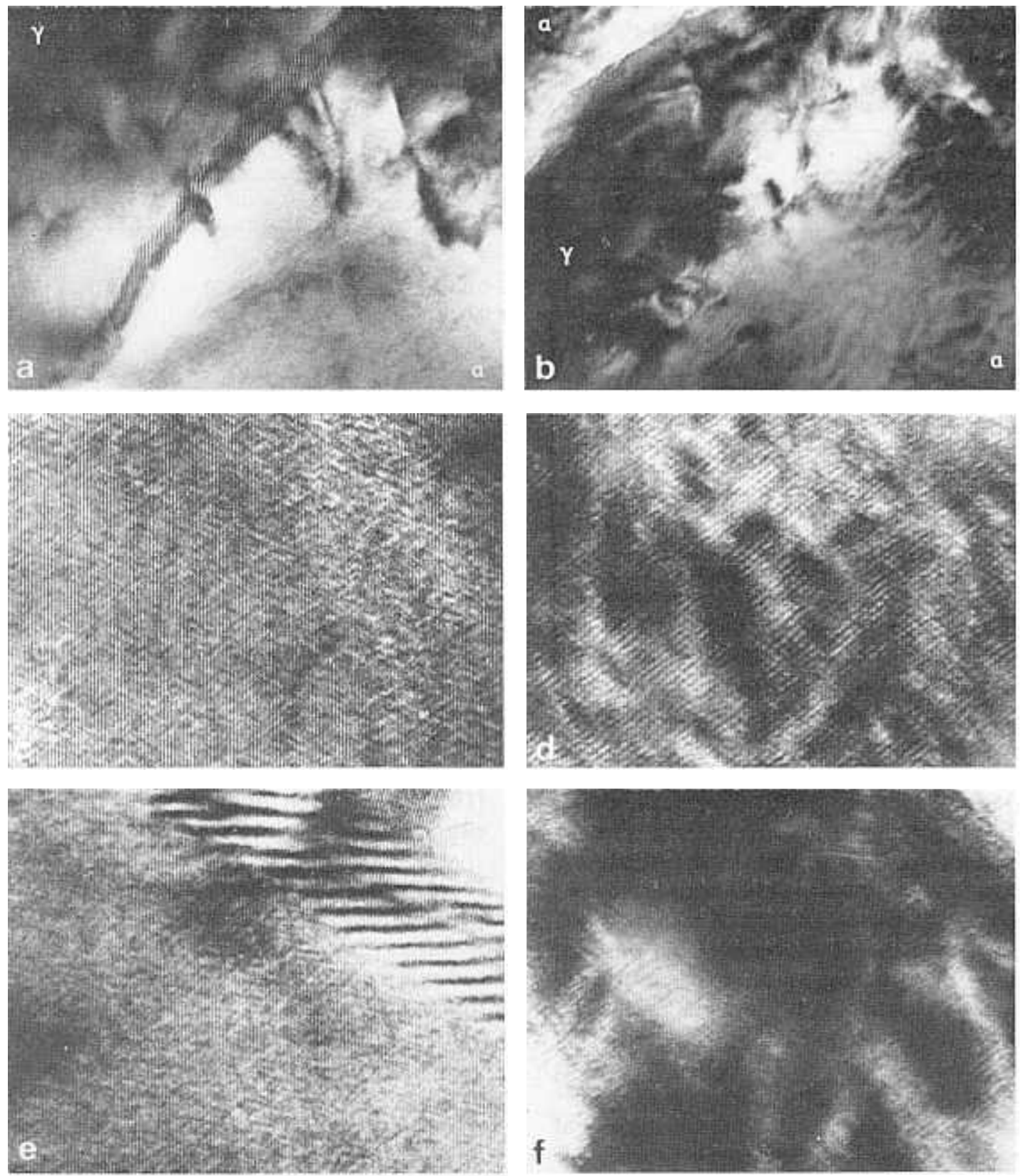

Fig. 5. Micrographs of the two steels used in section 4 showing fringes from the ferrite (c and d) and austenite (e and f). Alloy 1 is on the left and alloy 2 on the right.

austenite carbon contents by using the relation given above. The best accuracy obtained for the ratio was approximately $0.1 \%$ which is much worse than might be expected given the measurement accuracy for a single micrograph but reflects the strong variation of fringe spacing with focus and the small sizes of detectfree regions. This is particularly the case in alloy 2 where it was very difficult to obtain accuracies better 
than $0.3 \%$ because of the high defect density in the retained austenite.

The results show a large variation from region to region. This cannot be explained in terms of any of the effects which can make the lattice fringe spacing different from that of the lattice as described in section 3. There must thus be a real variation of the $\gamma$ lattice spacing from region to region. The displacive nature of the bainitic transformation does not allow substitutional alloying element partitioning, so the region variations cannot be linked with substitutional element segregation. While atom probe results (Bhadeshia and Waugh [24]) confirm that there is no substitutional element partitioning, the changes in lattice parameter that could be expected, even for gross $\mathrm{Mn}$ or Ni segregation, are anyway insignificant (see Pearson [21]).

It is thus clear that two predictions of the theory discussed above have been demonstrated:

(1) the overall carbon content of the austenite in alloy 2 is generally higher than that in alloy 1 ;

(2) in both cases the carbon is distributed very inhomogeneously. Furthermore, all but one of the results fall within the predicted $x_{\gamma}\left(T_{0}\right)$ to $x_{\gamma}\left(A e_{3}^{\prime}\right)$ range; the exception is not understood.

\section{Conclusions}

We have demonstrated that a lattice fringe spacing on a single micrograph of a typical uniform, defectfree material can be measured to an accuracy of $0.01 \%$ or better using either microdensitometric or optical diffraction techniques. We have also shown the usefulness of moire methods for the detection of suitable regions for more accurate measurements. Of more interest we have delineated the extent to which objective defocus can cause variation in the observed lattice spacing to a different degree under various observational circumstances, and show how this can be useful in obtaining a realistic value for the lattice parameters of a uniform material.

We have also considered how lattice fringe spacings can be related to the variation of a lattice parameter as a function of regular segregation as occurs during spinodal decomposition. We conclude that the variation in structure factor from point to point is as important as the variability of lattice spacing in deter- mining the lattice fringe to lattice spacing relationship and suggest ways of experimentally investigating this effect.

Our experiments on the measurement of lattice fringe spacings in steels containing retained austenite had the dual aim of examining the evidence for carbon segregation and testing the accuracy of the methods described on a system with a high dislocation content for which accuracies might be expected to be low. Nevertheless we were able to demonstrate that carbon segregation affects the lattice parameter sufficiently to test both thermodynamic models for the upper bainitic transformation and to discover real non-uniform carbon segregation.

\section{Acknowledgements}

The authors are grateful to Dr. A. Howie for discussion of some of the theory and to Dr. R.A. Ricks for supplying the micrographs used in section 3. Financial support from the Science Research Council is also acknowledged.

\section{References}

[1] M.A. O'Keefe and P. Buseck, Trans. ACA 15 (1979) 27.

[2] L.A. Bursill, J.G. Barry and P.R.W. Hudson, Phil. Mag. A37 (1978) 789.

[3] G.R. Anstis, D.F. Lynch, A.F. Moodie and M.A. O'Keefe, Acta Cryst. A29 (1973) 138.

[4] W.O. Saxton, J. Microsc. Spectrosc. Electron., in press.

[5] R. Sinclair and G. Thomas, Met. Trans. 9A (1978) 373.

[6] R. Sinclair, in: Proc. 9th Intern. Congr. on Electron Microscopy, Toronto, 1978, Vol. III, p. 140.

[7] Roier, Acta Cryst. A25 (1969) 516.

[8] J.W. Steeds, in: Introduction to Analytical Electron Diffraction, Eds. J.J. Hren, J.I. Goldstein and D.C. Joy (Plenum, New York, 1979).

[9] W.O. Saxton, T.J. Pitt and M. Horner, Ultramicroscopy 4 (1979) 343.

[10] C.K. Wu, R. Sinclair and G. Thomas, Met. Trans. 9A (1978) 381 .

[11] T. Mulvey, J. Microsc. 98 (1973) 232.

[12] J.C.H. Spence, J.M. Cowley and R. Gronsky, Ultramicroscopy 4 (1979) 429.

[13] R. Gronsky and G. Thomas, in: Proc. Modulated Structures Conf., Kailva Kona, Hawaii (Am. Inst. Phys., New York, 1979).

[14] J.W. Cahn and J.E. Hilliard, Acta Met. 19 (19710 151.

[15] J.S. Langer, Ann. Phys. (NY) 65 (1971) 53. 
[16] R.F. Cook and A. Howie, Phil. Mag. 20 (1969) 641.

[17] A. Howie, private communication (1980).

[18] H.K.D.H. Bhadeshia; Ph.D. Thesis, University of Cambridge (1979).

[19] H.K.D.H. Bhadeshia and D.V. Edmonds, Met. Trans. 10A (1979) 895.

[20] H.K.D.H. Bhadeshia and D.V. Edmonds, Acta Met. 28 (1980) 1265.
[21] W.B. Pearson, A Handbook of Spacings and Structures of Metals and Alloys, Vol. 2 (Pergamon, Oxford, 1967).

[22] C.S. Roberts, Trans. AIME 197 (1953) 203.

[23] N. Ridley, H. Stuart and L. Zwell, Trans. AIME 245 (1969) 1834.

[24] H.K.D.H. Bhadeshia and R. Waugh, unpublished. 